case of epilepsy, from some cause which is not obvious and remediable, does not make good progress under potassium, to try strontium. There are some things that nothing short of actual experiment will decide, and among them is the question, Which salt will best suit a given case of epilepsy?

$$
1 \text { Lancet, Ang. } 12 .
$$

\section{SPONTANEOUS FRACTURE IN PHOSPHORUS WORKERS.}

DR.W.F. DEARDEN ${ }^{1}$ draws attention to the occurrence of fragilitas ossium in workers among phosphorus. $\mathrm{He}$ says that although, in considering the dangers arising from the use of yellow phosphorus in the manufacture of lucifer matches, prominence has always been given to the liability to necrosis of the jaw, this is not the only important pathological condition incidental to this industry, there being grounds for believing that a certain condition is brought about in the bony tissue as a result of which fractures of the long bones are very easily produced. He gives cases illustrating the condition, and refers to the recently issued report to the Home Secretary on the Use of Phosphorus in the Manufacture of Lucifer Matches, in which a special note is made of the experience of Dr. Boocoorens in Belgium, who "met with and treated in twenty-three years 30 cases of spontaneous fracture of the long bones caused by muscular effort, affecting exclusively the lower limbs of workmen who had been employed for years in dangerous departments and who had also suffered from necrosis of the jaw." The same sort of thing has also been reported by Dr. Kocher, of Berne, and Dr. Garman, of Bow Road.

' Brit. Med. Jour., July 29.

\title{
ProGress IN MEDICINE.
}

\section{DISEASES OF THE BLOOD.}

(Continued from page 330 .)

Examination of the Blood.-Louis Fenner ${ }^{12}$ recommends a simple solution which fixes and double-stains blood films in two minutes and keeps well. It is derived from the precipitate formed in a watery solution of equal parts of eosin and methylene blue. The author takes Grübler's water soluble eosin (yellow shade) and methylene blue, dissolves them separately in distilled water and mixes the solutions in an open dish. The precipitate is washed and dried twice, and finally dissolved ( 5 grm. in 100 c.c.) in methyl alcohol. Cover glass preparations are simply dried and have a few drops of this solution poured on them. They are placed under a watch glass to prevent evaporation for one to three minutes, then rinsed in distilled water for about eight seconds, lightly dried, and mounted in xylol balsam. Red corpuscles appear of a terra-cotta colour, nuclei, blue; granules of basophil cells, violet; those of the eosinophils red; and bacteria or plasmodes, blue. The colouring matter is thus split up, and the acid tissues combine with the basic part and vice versa. It is essential that the coverslips should be free from any acid or alkali; and to this end, after washing in distilled water, they must be kept in absolute alcohol before using, and distilled water only should be employed in rinsing the specimens. The dried staining material can be obtained ready for use from Kanthack, 18, Berners Street. P. N. Gerard ${ }^{13}$ uses the Aronson and Phillips' stain for malaria undiluted, and fixes the specimens by drying them at 120 deg. C. for two minutes. They are then placed in the strong stain for two minutes only, and thoroughly washed till part of the film shows an orange tint. The corpuscles are a very bright orange, and parasites, though almost colourless, show well by contrast. In counting white and red corpuscles A. G. Phear ${ }^{14}$ uses a single liquid film, and in order to increase the area needed for a sufficient number of white cells he employs the camera lucida, marking out the squares on a piece of cardboard by the side of the microscope. The image of the cells lying over the unruled part of the stage is thrown on to these squares in succession, and their number counted. As a diluting fluid he finds Sherrington's formula gives good results, preserving the colour and shape of the red cells, and staining the nuclei of the white ones. It is made as follows: Distilled water, 300 grammes ; sodium chloride, 1.2 ; neutral potass. oxalate, 1.2 ; methylene blue, 1.0 ; mix.

Addison's Disease.-A. G. Auld ${ }^{15}$ upholds his original theory that the medulla of the suprarenals is the only active part, and quotes Abel's result's in favour of the active principle being an alkaloid with the formula $\mathrm{Cl}_{17} \mathrm{H}_{15} \mathrm{NO}_{4}$. From recent experiments he argues that this substance, if injected, is destroyed in the blood and organs. Besides, when one suprarenal gland is removed, he finds the spleen becomes enlarged, and the thymus hypertrophied. If both are taken away death follows in one or two days. He concludes that the substance in question is not a secretion, but a poison separated from the blood by the gland, and as a result of its decomposition a vasoconstricting material may be formed in the gland, but not excreted as such. The extraordinary power of the extract of suprarenals in contracting blood-vessels is attested by various writers. ${ }^{16}$ It has been used in the eye and on granulating wounds as an astringent, while it has no effect on accommodation. In chloral and chloroform poisoning intravenous injections at once cause recovery in lower animals. It has, however, little effect when given by the mouth, or injected into the subcutaneous tissues, on account of its rapid elimination or destruction. It has been used, however, externally in recent eczema, and, according to several observers, it possesses a marvellous power of stopping the symptoms of hay fever for twelve hours or longer. Dreyer argues that it is a secretion of the gland, and can be found in the adrenal vein; while, as we have seen, Abel ${ }^{17}$ has isolated it, determined its percentage composition, and named it Epinephrin. The free base prepared at low pressure, as well as its sulphates and benzoates, is active in producing the effects of the crude gland extract. It is definitely shown to be distinct from pyrocatechin, and now that it can be obtained pure widely extended trials may be looked for, as well as its synthetic preparation. It has been estimated that the glands of 2,000 sheep only contain one drachm of pure Epinephrin, so that a means of synthetic formation is much to be desired. ${ }^{18}$ Its formula is similar to sanguinarine, which also possesses the power of raising the blood pressure.

Bates $^{19}$ has employed suprarenal extract in dry catarrh of the ear, and for tinnitus due to inflammatory condi- 
tions. He quotes also a case of pernicious anæmia where the red cells were markedly increased, and one of Graves' disease under the care of Crary which improved in every respect under its use. Wallace and Mogt ${ }^{20}$ found that the extract stimulates the vagus and also the heart muscle directly, as well as producing a marked contraction in the arterioles of the systemic circulation, but not in those of the pulmonary vessels. Destot treated a case of Addison's disease with increasing injections of the extract, and found that the pigmentation rapidly disappeared. The treatment, however, had to be stopped from the condition of the bowels. Carlin Philips ${ }^{21}$ discusses a case of the disease where the suprarenals proved to be atrophied, which has been noted in 14 other cases, and, indeed, appears to be the most common cause of the disease apart from tuberculosis. However, J. Levin points out that in 12 per cent. of the cases of Addison's disease the suprarenals are quite normal, so that the connection between the two conditions is uncertain. A well-marked case of the disease in a woman of 38 , treated by the extract, is reported by A. Foster. ${ }^{22}$ Some relief was noticed, the pulse was quickened, and the pain in the back diminished, and there was less faintness; but the pigmentation remained, and the patient died after a series of convulsions. This temporary improvement has been noticed in some like cases. Others, according to G. Ringer and Phear, are much benefited, while in most instances very little effect is shown. ${ }^{23}$ In a patient under the care of Charlewood Turner, ${ }^{24}$ where the extract was tried too late to be of service, the blood pressure was measured by the sphygmometer, and the reduction produced by the disease was proved to be great. Instead of being equal to 100 or $125 \mathrm{~mm}$. of mercury, as in health, the tension was only $73 \mathrm{~mm}$. An occasional termination of Addison's disease is insanity. Munson some time ago reported two cases in which depression, dulness, and groundless fears were noticed, and irritability and violence came on later. A. Mclane Hamilton ${ }^{25}$ speaks of another where the disease developed in a weakminded man, and was followed by depression, delusions, and angry outbursts. He refers to two other instances of a like nature, and, in discussing the value of the extract, mentions that he has given it with some success in exophthalmia; while in two cases of hysterical mania it reduced the excitement and caused sleep when 5 to 20 grains were administered in divided doses. Thibierge ${ }^{: 6}$ showed recently an instance of Addison's disease in a negro. The mucous membrane of the mouth was marked by numerous patches of pigment, and the skin had become definitely darker during the last three years. The patient was tuberculous, and complained of lumbar pains and great weakness, as is usually the case.

Acromegaly.-Lyon ${ }^{27}$ records the treatment of a fat, apathetic patient with Hypophysin. The pulse became regular, the headache, nystagmus, and mental condition were improved, and the weight was reduced by nine kilogrammes. Leszynsky ${ }^{23}$ showed a well marked case of the disease in a policeman aged 36 , with failing vision, alterations in the visual field, loss of smell and sexual power, and enlargement of phalanges, feet, clavicles, and bones of the head. Oliver Osborne remarked that these cases could be distinguished from pulmonary osteo-arthropathy by the hands alone, for in the latter the fingers are sausage shaped and the nails small. He denied that a large thymus exists in acromegaly, the mass so often found being really a thoracic enlargement of the thyroid gland. Other observers have noticed that the thyroid is enlarged in a number of instances, while the thymus, or what was taken for it, is recorded as persisting in eight patients. The heart, the liver, and spleen are frequently found of abnormal'size. An elaborate review of the literature of the subject is given by Mitchell and LeCount, ${ }^{29}$ who conclude that true tumours of the hypophysis are not so numerous as has been thought, and the disease does not entirely depend on the loss of function of that organ. The changes in the hypophysis may, they think, be sometimes due to enlargement of the sella turcica, and the cases examined show that some connexion exists between the thyroid and hypophysis. The enlargement of the heart, thyroid and sella turcica is more constant than pathological changes in the hypophysis.

12 Lancet, Feb. 11. 13 Lancet, June 3. 1s Lancet, May 20. 15 Brit. Med. Jour., Jan. 3. ${ }^{16}$ Merck's Archives, Feb. 17 Brit. Med. Jour., Mar. 3. 18 Oolumbus Med. Jour., Mar. 3. 19 Les Nouveanx Remodes, April 8. ${ }^{20}$ Brit. Med. Jour., Mar. 3. ${ }^{2}$ 'Med. Rec., Mar. 18. ${ }^{22}$ Lancet, June 10. 23 Transact. Olin. Soc., Vol. xxix., p. 68. " Lancet, June 10. ${ }^{25}$ Med. Rec., April 29. ${ }^{26}$ New York Med. Jour., Mar. $25 .{ }^{27}$ Brit. Med. Jour.,
Dec. 10. ${ }^{28}$ Med. Rec., Mar. 4. 29 New York Med. Jour., April 15, 22, 29.

\section{Progress IN SURGERY.}

TUMOURS.

(Continued from page 332.)

Cases of Special Interest.-W. H. Bennett ${ }^{9}$ has pub. lished a series of cases illustrating peculiarities in the behaviour of tumours. Some illustrate the occurrence of tumours apparently malignant which ran an innocent course ; others are examples of tumours apparently innocent proving in the end to be malignant; and a third group illustrates the effect of concentration of the mind in the production of tumours innocent or malignant. A few of these may be briefly described. Case 1: A woman of 36 had in seven months become weak, greatly emaciated, and sallow, and had developed a tumour in the abdomen under the liver. The growth on opening the abdomen was found to be a nodulated mass of stony hardness, and appeared to invade the liver. On the upper surface of the latter was a separate nodule the size of half a walnut. The omentum was involved. A small piece was examined microscopically, and pronounced to be mixed round a spindle-celled sarcoma. Eighteen months later she was a wellnourished woman, and there was no trace of tumour. Case 2: A woman of 52 had a well-marked melanotic sarcoma of the sole of the foot excised. Later in the same year Syme's amputation was performed for recurrence. A month later there were some blackish tumours in the skin on the inner side of the thigh. These multiplied until 62 could be counted. There were at the same time hard glands in Scarpa's triangle. The primary and secondary growths were proved to be melanotic sarcomata microscopically. The case was regarded as hopeless. Subsequently all these nodules 